\title{
Capacity Optimization of MIMO Systems Involving Conformal Antenna Arrays using a Search Group Algorithm
}

\author{
Abdelmadjid RECIOUI \\ Laboratory of Signals and Systems, Institute of Electrical and Electronic Engineering \\ University M'hamed Bougara of Boumerdes \\ Boumerdes, Algeria. \\ a recioui@univ-boumerdes.dz
}

\begin{abstract}
MIMO systems constitute a backbone of the fourth and fifth generations of wireless communication systems. The purpose of this paper is to introduce the involvement of conformal antenna arrays into MIMO systems. The Search Group Algorithm (SGA) is then used to further enhance the capacity of MIMO system employing conformal antenna arrays at both ends (Transmitter; Tx and Receiver; Rx). The results reveal that compared to the linear and 2D cases, conformal antenna arrays promise higher capacity values which motivates their employment in future MIMO communication systems.
\end{abstract}

Keywords: MIMO systems, Capacity enhancement, Search Group Algorithm, conformal antenna arrays.

\section{INTRODUCTION}

MIMO techniques constitute an area of interest in nowadays wireless communication. The employment of multiple antennas leads in a significant improvement in capacity without any additional allocated power or bandwidth [1]. MIMO systems exploit the multipath structure of the propagation channel. However, correlations among channel coefficients are influenced by the antenna properties. As the antennas are collocated in a MIMO array, mutual coupling effects may occur [2]. All these effects should be considered when designing an antenna array for MIMO systems.

Many MIMO antenna design optimization studies have been reported in literature. Capacity formulas are given in [3] and [4] for mutual coupling and spatial correlation effects with a Rayleigh fading channel being assumed. In addition, the geometries considered constitute namely uniform linear arrays with spacing between the elements being optimized. In [5] and [6], the issues of how can designers appropriately select the number of antennas at the asymmetric base station and mobile units have been addressed. In [7], optimizing the MIMO system capacity with unequal costs of implementing antennas at both channel ends has been dealt with. However; in this work, the cost function is expressed using approximated asymptotic expression for the ergodic capacity calculations.
From geometry selection point of view, Uniform Linear Array (ULA) is the most common geometry in modern wireless systems. The Uniform Circular array (UCA) is as an alternative geometry with some enhanced properties. The results in [8-10] show that the spatial correlation decreases for UCA compared to ULA on average for small and moderate Angular Spread (AS) for similar aperture sizes. On the other hand, ULA has less spatial correlation than UCA for near broadside angle-of-arrivals with moderate AS. Recioui and Bentarzi [11] challenged capacity maximization through optimizing the spacings between the elements of a linear array taking into account mutual coupling and spatial correlation simultaneously. A channel model was proposed to account for both mutual coupling and spatial correlation and has been validated through comparing it with its independent counterparts. The results reveal an enhancement in system capacity relative to the approaches reported in literature which can be advantageous in modern wireless communication systems. The geometry considered in that work was a linear array of half wave dipoles arranged in a side-by-side configuration at both transmitter and receiver. Thus far, methods based on the genetic algorithm, ant colony optimization, particle swarm optimization, Tabu search, bees' algorithm, differential evolution, and Clonal selection have become more popular, and they have been used in solving antenna array 
pattern synthesis problems. The performances of these methods are found to be better than those of the classical optimization techniques and the conventional analytical techniques. Each of these methods has its specific advantages and disadvantages. Evolutionary optimization techniques that are effective in solving multimodal function optimization problems have also been applied in antenna design. In this work, the capacity of MIMO system is attempted to be maximized using a Search Group optimization algorithm. The extension of the employment of linear arrays at both MIMO system ends to the conformal array deployment is considered. The idea is to consider considering mutual coupling for the independent and identically distributed (i.i.d.) flat Rayleigh fading channel model, assuming uniform transmit power allocation schemes. To find the maximum capacity achievable for MIMO system, an optimization process is carried out to find the optimal physical spacings that produce this desired capacity.

\section{PROBLEM FORMULATION}

The capacity of a MIMO channel system is given as the maximum data transfer rate for a given acceptable level of received signal and is given in bps/Hz (bits per second per hertz). In its general form, the capacity of a MIMO channel having $N_{t}$ transmitting antennas and $N_{r}$ receiving antennas assuming equal signal power is given as [13]:

$$
\text { Cap }=\log _{2}\left[\operatorname{det}\left(I_{N_{r}}+\frac{S N R}{N_{t}} H H^{H}\right)\right]
$$

Where $I_{N_{r}}$ is an $N_{r} \times N_{r}$ identity matrix, $S N R$ is the average received signal to noise ratio in $\mathrm{dB}, H$ is the channel transfer matrix and the $H$ on the exponent is the complex conjugate transpose.

Since the channel matrix is random, the capacity of the MIMO channel is a random variable. The capacity of fading channels can be defined in a number of ways. In practice, mean capacity and outage capacity are the two most commonly used statistical measures. The mean capacity of a MIMO channel is the ensemble average of the information rate over the all realizations of the channel matrix [13]. The meaning of the mean capacity is that in an ergodic channel, we can transmit the signal at the rate given by mean capacity without errors. This representation has been adopted in the present work.
To take into account the mutual coupling effect, the channel transfer matrix is written as [14-15]:

$$
H_{M C}=C_{R} G C_{T}
$$

Where $\mathrm{G}$ is again an $N_{r} \times N_{t}$ matrix with entries identically distributed (i.i.d) complex Gaussian zero-mean unit variance elements. $C_{R}$ and $C_{T}$ are the coupling matrices at the receiver and transmitter, respectively. These matrices are computed as:

$$
C=\left(Z_{A}+Z_{T}\right)\left(Z+Z_{T} I_{N}\right)^{-1}
$$

Where $Z_{A}$ is the self impedance of the element in isolation $\left(Z_{A}=73+42.5 \mathrm{j}\right.$ for a $\frac{\lambda}{2}$

dipole; $\lambda$ being the wavelength). $Z_{T}$ is the impedance of the receiver at each antenna element chosen to be the complex conjugate of $Z_{A}$ to obtain an impedance match for maximum power transfer. $I_{N}$ is the identity matrix and $Z$ is an $N \times N$ mutual impedance matrix.

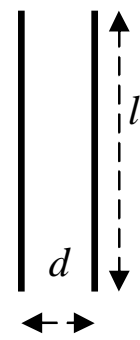

(a)

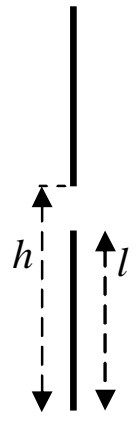

(b)

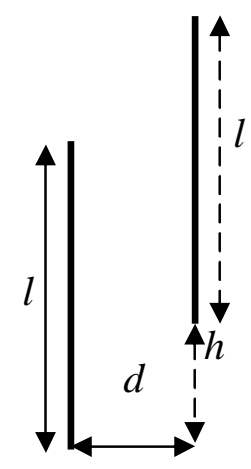

(c)
Fig. 1 Different configurations for linear array of dipoles used at either Tx or Rx with their physical parameters (shown here are two elements)

(a) Side-by-side configuration

(b) Collinear configuration

(c) Parrallel-in-echelon configuration

The channel matrix needs to be properly normalized [15]. There exist two normalizations:

The first normalization is performed on the each realization of the end-to-end channel gain as well as mutual coupling matrix $H_{M C}$, such that [15]

$$
\left\|H_{M C}\right\|_{F}^{2}=N_{R} N_{T}
$$

Where $\|\cdot\|_{F}^{2}$ is the Frobenius norm. The limitation of this normalization is that the 
differences in the channel gain due to antennas are removed. However this type of normalization permits the investigation of the correlation between the channel matrix entries and gives good indication of the richness of the multipath environment [1415].

The second normalization is summarized by the equation:

$$
\|G\|_{F}^{2}=N_{R} N_{T}
$$

This normalization is also performed on each realization of the channel matrix, but includes the propagation channel only. This normalization permits investigation of the effects of instantaneous changes of the received power due to mutual coupling. In this work, since the geometrical dimensions of the various array geometries constitute the main concern; the first normalization has been used.

A conformal array is an array that conforms to some geometry. It may be regarded as a combination of individual linear arrays. In this work, two geometries are considered: the cylindrical and the conical shapes. The mutual impedance matrices presented earlier for the different linear array configurations can be extended to the conformal arrays. The elements of the conformal array exhibit the three configurations at the same time. An element may be in side-by-side configuration with the adjacent element, in collinear configuration with the element below and in parallel-in-echelon with the diagonal element. Each element exhibits mutual coupling with all the other elements in the 3D array. This turns out to produce a mutual impedance matrix with size equal to $27 \times 27$ in this case. In general, an array with row elements $N$ and column elements $M$ results in a coupling matrix of size $M N \times M N$. The entries of the impedance matrix are computed based on the equations previously presented depending on the relative configurations of the elements. The general representation of the impedance matrix is given as:

$$
Z=\left[\begin{array}{lll}
Z_{11} & Z_{12} & Z_{13} \\
Z_{21} & Z_{22} & Z_{23} \\
Z_{31} & Z_{32} & Z_{33}
\end{array}\right]
$$

Where the entries given above should be regarded as $3 \times 3$ block matrices. The matrix is symmetrical about its diagonal block elements. The diagonal block elements represent the mutual impedance matrices within the horizontal arrays. As an example,
$Z_{12}$ is the $3 \times 3$ impedance matrix due to the array 1 and array 2 . The diagonal elements of this matrix represent the mutual impedance due to the elements in array 2 with those just above them. These are in collinear configuration. The other entries are computed using the parallel-in-echelon configuration. Finally, the other blocks are just constructed by symmetry.

Once the impedance matrix is computed, the coupling matrix is found using (3) and hence the system capacity is obtained using (1) and (2).

\section{SEARCH GROUP ALGORITHM (SGA)}

Search group algorithm (SGA) is a relatively new population-based optimization technique derived by Howlader et al. in 2015[20]. The basic idea of SGA is to hold a natural balance between exploration \& exploitation while discovering the global optimum solution. At first iteration, SGA tries to explore the most promising search space within the defined search boundary, and then SGA refines the generated solutions as iterations processed. The operation of SGA can be explained by dividing its operations into five steps, such as initialization, selection of search group, mutation, creation of family in each search group, \& selection of new search group [20].

\section{A. Initialization}

Like other evolutionary algorithms (EAs), SGA initializes the population randomly within the defined search area. To generate initial population [20].

$$
\begin{aligned}
& \text { for } i=1: n_{p} \\
& \qquad \begin{array}{l}
\text { for } j=1: \operatorname{dim} \\
\quad P_{i j}=x_{j}^{\text {min }}+\text { rand }^{*}\left(x_{j}^{\max }-x_{j}^{\text {min }}\right) \text {; }
\end{array} \\
& \text { end } \\
& \text { end }
\end{aligned}
$$

where $n_{p}$ is population size; dim indicates the dimension of control parameters;

$x_{j}^{\min } \& x_{j}^{\max }$ are minimum \& maximum values of $j^{\text {th }}$ control variable; $P_{i j}$ is population of $j^{\text {th }}$ control variable in $i^{\text {th }}$ iteration.

\section{B. Selection Of Search Group}

After initialization, the fitness value of individual generation has been calculated \& best solutions among them are marked as 
elite solutions. Based on the solutions, a search group ' $S$ ' is constituted by following a standard tournament process [20].

\section{Mutation}

To improve the global search ability, mutation phase is performed by using (7) [20]:

$$
x_{j}^{m u t}=E\left[S_{:, j}\right]+t \varepsilon \sigma\left[S_{:, j}\right]
$$

where $x_{j}^{m u t}$ indicates $j^{\text {th }}$ design variable of a given mutated individual; $E \& \sigma$ are mean \& standard deviation, respectively; $\varepsilon$ indicates the mutation variable; $t$ controls how far a mutated individual is generated from the mean value of the population; $S_{:, j}$ is $j^{\text {th }}$ column of search group matrix.

\section{Generation of Families in Each Search Group}

The families are set including members of search group \& the individual generated. Each members of the generated search family is perturbed by using (8) [20].

$$
x_{j}^{\text {new }}=S_{i j}+\alpha \varepsilon
$$

where $\alpha$ is control size of perturbation. It helps SGA to control the exploration phase. The value of $\alpha$ is updated by using (9)[20].

$$
\alpha^{l+1}=b \alpha^{l}
$$

where $b$ is parameter of SGA; $\alpha^{l}$ it controls the distance that a new individual is generated from its search group member.

\section{E. Selection of New Search Group}

SGA is comprised of both global \& local phases. In the first iteration of iter $_{\text {max }}$, SGA explore most of the promising search space so as to form a search group including members of the individual family. When the current iteration is larger than iter $_{\text {max }}$, SGA forms a search group with best solutions for all families. The latter phase is called 'local phase' because in this phase SGA exploits the area of the current optimization space. SGA is different from the other algorithms in what follows.

- The optimization strategy is based on the computation of mean and standard deviation of the position of current search group members in a given iteration.

- The SGA involves local \& global phases to update the members in a search group.
The proposed SGA requires prior initialization of some algorithm specific control parameters, these are: $\alpha^{l}$ (controls both exploration and exploitation phases) $\in(0.75,2.25) ; b$ defines the manner that $\alpha^{l}$ decreases during the course of a generation; $n_{\text {mut }}$ (number of mutations at each iteration): $3 \%$ of the population; $n_{g}$ (number of search group members): $20 \%$ of the population. The general flowchart of SGA is shown in Fig. 2. For more details on SGA, readers are referred to [20].

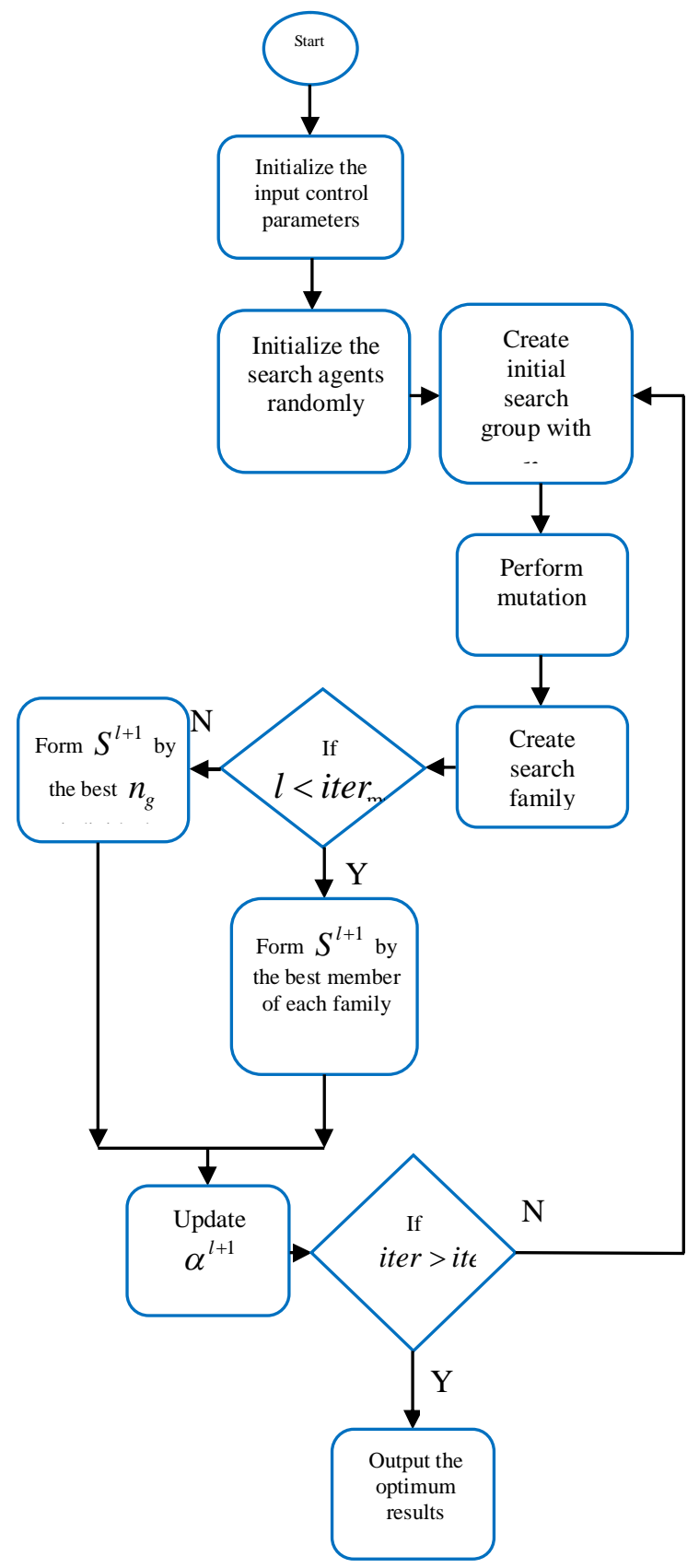

Fig. 2 Flowchart of SGA 


\section{RESULTS AND DISCUSSIONS}

The work deals with MIMO systems of different sizes with conformal antenna arrays at Tx and Rx. Cylyndrical and conical array geometries are considered. The average SNR is taken to be $20 \mathrm{~dB}$ for all cases. Regarding array inter-element spacing intervals, the horizontal spacing (d) spacing and the vertical spacing $(h)$ are constrained to range from 0.25 to 2 wavelengths. The linear arrays are optimized based on the same philosophy as the work in [19]. The results obtained with rectangular array antennas are compared to the values optimized when optimizing linear arrays in side-by-side configuration, uniform rectangular arrays with half wavelength spacing horizontally and vertically. Table 1 below presents the obtained capacity values.

Table 1 Summary of the obtained results and the cases treated

\begin{tabular}{|c|c|c|c|c|}
\hline $\begin{array}{c}\text { System } \\
\mathrm{N}^{\circ}\end{array}$ & $\begin{array}{c}\text { Type of } \\
\text { arrays at } \\
\text { ends }\end{array}$ & Dimensions & $\begin{array}{c}\text { Optimized } \\
\text { capacity } \\
\text { (bps/Hz) }\end{array}$ & $\begin{array}{c}\text { Capacity } \\
\text { for } \\
\text { Uniform } \\
\text { linear } \\
\text { arrays }\end{array}$ \\
\hline 1 & $\begin{array}{c}\text { Tx:Cylindrical } \\
\text { Rx: } \\
\text { Cylindrical }\end{array}$ & $\begin{array}{c}\text { Tx:18 } \\
\text { Rx: } 18\end{array}$ & 29.12 & 25.13 \\
\hline 2 & $\begin{array}{c}\text { Tx:Cylindrical } \\
\text { Rx: Conical }\end{array}$ & $\begin{array}{c}\text { Tx:18 } \\
\text { Rx: } 24\end{array}$ & 30.16 & 25.41 \\
\hline 3 & $\begin{array}{c}\text { Tx:Conical } \\
\text { Rx: } \\
\text { Cylindrical }\end{array}$ & $\begin{array}{c}\text { Tx:24 } \\
\text { Rx: } 18\end{array}$ & 31.39 & 26.45 \\
\hline 4 & $\begin{array}{c}\text { Tx:Conical } \\
\text { Rx: Conical }\end{array}$ & $\begin{array}{c}\text { Tx:24 } \\
\text { Rx: } 24\end{array}$ & 32.41 & 26.51 \\
\hline
\end{tabular}

A general remark about the results in Table 1 is that the capacities obtained using the Search Group optimization technique are better than the state of art ones when using uniform linear arrays.

Many examples have been treated to assess whether the employment of conformal arrays at both MIMO ends would enhance the system capacity. In the first example, 18element cylindrical arrays are employed at both $T x$ and $R x$. The results reveal an enhancement in capacity relative to both uniform an nonuniform linear arrays. Indeed, the employment of conformal arrays has led to a capacity increase of about $7.2 \%$ relative to the uniform linear array with one dimension larger.

In the second example, an 18-element cylindrical array has been used at the transmitter with a 24-element conical one at the receiver. The results show better capacity compared to the employment of 3-element linear arrays at both ends. The capacity increase is found to be $8.7 \%$ relative to the uniform array. A better enhancement would have been attained if just 2 element arrays have been employed.

As a third example, the opposite scenario to example 2 is treated. This case produced a better capacity enhancement of $10 \%$ compared to the employment of 3dimensional uniform linear arrays.

In the last example, two 24-element conical arrays are employed at both ends. This produces a capacity value that is $7 \%$ more than when using a 4-dimensional uniform linear array and $45.85 \%$ relative to using 3element uniform linear arrays.

It is to be noted that in the linear array case, only the horizontal dimension is given as the only geometry considered is the side-by-side configuration. As a further measure of practicability, the spaces occupied by the arrays are also provided. This spacing is given in wavelengths for the linear arrays and in squared wavelengths for the rectangular arrays.

\section{CONCLUSION}

The problem of optimizing the capacity of wireless communication systems employing conformal antenna arrays at both ends of the communication channel has been addressed. The purpose was to find the best interspacing dimensions that lead to maximum MIMO system capacity. The results have shown promising enhancement in capacity as compared to the conventional uniform antenna arrays. Particularly, the employment of conformal antenna arrays at both transmitter and receiver is recommended as higher capacity values are obtained.

\section{References}

[1] F. Farrokhi, A. Lozano, G. Foschini and R. A. Valenzuela, "Spectral efficiency of FDMA/TDMA Wireless systems with Transmit and Receive Antenna Arrays", IEEE Trans. On Wireless Comm., Vol.1, Oct. 2002.

[2] Constantine A. BALANIS, "Antenna theory: analysis and design" Third edition, John Wiley \& Sons Inc., 2005.

[3] S. Durrani and M. E. Bialkowski, "Effect of mutual coupling on the interference rejection capabilities of linear and circular arrays in CDMA systems," IEEE Trans. Antennas Propagat., vol. 52, no. 4, pp. 1130-1134, Apr. 2004.

[4] Daniele Piazza, Nicholas J. Kirsch, Antonio Forenza, Robert W. Heath and Kapil R. Dandekar, "Design and evaluation of a reconfigurable antenna array for MIMO systems", IEEE trans. Anten. Propag., Vol. 56, No. 3, March 2008. 
[5] Lozano, A. and A. M. Tulino, "Capacity of multipletransmit multiple receive antenna architectures," IEEE Trans. Inf. Theory, Vol. 48, No. 12, 3117-3127, Dec. 2002.

[6] Oyman, O., R. U. Nabar, H. Bolcskei, and A. J. Paulraj, "Tight lower bounds on the ergodic capacity of Rayleigh fading MIMO channels," Proc. GLOBECOM, 1172-1176, Taipei, Taiwan, R.O.C., Nov. 2002.

[7] $\mathrm{Du}, \mathrm{J}$. and Y. Li, "Optimization of antenna configuration for MIMO systems," IEEE transactions on Communications, Vol. 53, No. 9, 1451-1454, Sept. 2005.

[8] Waheed, U. A. and D. V. Kishore, "Uplink spatial fading correlation of MIMO channel," 58th IEEE Vehicular Technology Conference, VTC 2003, Vol. 1, 94-98, 2003.

[9] Tsai, J.-A. and B. D. Woerner, "The fading correlation of a circular antenna array in mobile radio enviroment," IEEE Global Telecommunications Conference, Vol. 5, 32323236, 2001.

[10] Xin, L. and Z.-P. Nie, "Spatial fading correlation of circular antenna arrays with laplacian PAS in MIMO channels," IEEE Antennas and Propagation Society International Symposium, Vol. 4, 3697-3700, 2004.

[11] A. Recioui and H. Bentarzi, "Genetic Algorithm based MIMO capacity enhancement in spatially correlated channels including Mutual Coupling", Vol. 63, No. 3 (2012), 689-701, DOI: 10.1007/s11277-010-0159-5

[12] A. Recioui, "Design and Thinning of linear and planar antenna arrays using a binary teaching learning optimizer", Acta Physica Polonica A, Vol. 130, Issue 1, 2016. pp: 7-8.

[13] G. J. Foschini and M. J. Gans, "On limits of wireless communications in a fading environment when using multiple antennas", Wireless Personal communications, Vol. 6, No. 3, pp 311-335, Mar. 1998.

[14] R. Janaswamy, "Effect of element mutual coupling on the capacity of fixed length linear arrays," IEEE Antennas Wireless Propagat. Lett., vol. 1, pp. 157-160, Oct. 2002.

[15] Shuo Pan, S. Durrani and M. E. Bialkowski, "MIMO Capacity for Spatial Channel Model Scenarios," Proc. Australian Communications Theory Workshop (AusCTW), Adelaide, Feb. 5-7, 2007, pp. 25-29.

[16] 3rd Generation Partnership Project (3GPP), "Spatial channel model for multiple input multiple output (MIMO) simulations (3gpp tr 25.996 version 6.1 .0 release 6)," ETSI, Tech. Rep., 2003.

[17] A. RECIOUI and H. BENTARZI, "MIMO system capacity enhancement in spatially correlated channels using Taguchi Method", $12^{\text {th }}$ WSEAS International conference on mathematical methods, computational techniques and intelligent systems in Sousse, Tunisia, 03 au 06 Mai 2010.
[18] Goncalves, M.S., Lopez, R.H., Miguel, L.F.F. (2015) Search group algorithm: A new metaheuristic method for the optimization of truss structures. Computers and Structures, 153, 165184. 